\title{
Focal Nodular Hyperplasia of the Liver Presenting as Right Upper Quadrant Mass
}

\author{
Adedapo Olumide Osinowo ${ }^{1}$, Olanrewaju S Balogun ${ }^{1}$, Thomas O Olajide ${ }^{1}$, Kabir B Badmos² \\ ${ }^{1}$ Department of Surgery, General Surgery Unit; ${ }^{2}$ Department of Anatomic and Molecular Pathology; College of Medicine, University \\ of Lagos and Lagos University Teaching Hospital, Surulere, Lagos.
}

Corresponding Author:

Dr. Adedapo Olumide Osinowo

Email: dapoosinowo20002000@yahoo.com

This is an Open Access article distributed under the terms of the Creative Commons Attribution License (creativecommons.org/ licenses/by/3.0).

Received

Accepted

May 3, 2018

Published

June 26, 2018

August 20, 2018

\begin{abstract}
Background: Focal nodular hyperplasia (FNH) is the second most common benign liver tumor, which in the vast majority of cases occurs in young women. The lesion is often discovered incidentally as a result of the widespread availability and application of modern cross-sectional imaging on the abdomen. We herein present a case of symptomatic FNH that had successful resection. Case Report: A 22-year old female patient with a history of recurrent right upper quadrant abdominal pain of 11 years duration was reviewed in our facility. Abdominal examination revealed a roughly mobile oval swelling in the right upper quadrant. Contrast abdominal computerized tomography scan revealed a mass isoattenuating in density relative to muscle, enhanced intravenous contrast and displacing bowel loops measuring $13.9 \times 8.3 \mathrm{~cm}$ in the transverse mesocolon. She had resection of the mass with an excellent post-operative quality of life. Pathological examination using Hematoxylin-eosin staining examination revealed focal nodular hyperplasia of the liver. Conclusion: Symptomatic focal nodular hyperplasia can be safely resected with excellent rates of morbidity and zero mortality.
\end{abstract}

Keywords: Abdomen, Abdominal Pain, Focal nodular hyperplasia, Liver Neoplasms, Tomography.

\section{Introduction}

Focal nodular hyperplasia (FNH) is the second most common benign liver tumor, which in the vast majority of cases occurs in young women [1]. The lesion is often discovered incidentally on cross-sectional imaging of the abdomen but can also present with vague abdominal symptoms due to mass effect causing pain and bloatedness. The contemporary management of FNH ranges from simple observation to major hepatic resection and the key determinants of clinical decision-making are symptomatic assessment and diagnostic certainty [2]. Our patient had a symptomatic presentation and the lesion was safely resected with excellent quality of life after surgery. The lack of malignant potential in FNH means that when relative diagnostic certainty is achieved, the asymptomatic patient might be safely managed conservatively [2] .
However, the relative diagnostic certainty should be undertaken with contrast-enhanced magnetic resonance imaging, which has been shown to be the most sensitive modality for characterizing this lesion.

\section{Case Report}

A 22-year old female patient with a history of recurrent right upper quadrant abdominal pain of 11 years duration was reviewed in our facility. The pain was dull in nature with no known aggravating or relieving factors. It was non-radiating and had no correlation with meals. Furthermore, it was not associated with nausea, anorexia, vomiting, change in bowel habit or jaundice. There were no genitourinary symptoms and the abdominal pain had no association with her menstrual cycle. She attained menarche at 11 years of age, has regular 
menstrual cycles and no dysmenorrhea. In addition, there were no associated respiratory, seizures, focal lateralizing signs or constitutional symptoms.

Her genotype is AA, has no history of blunt abdominal trauma and does not use oral contraceptive pills. She is the first born in a family of four children and there are no similar complaints in other siblings. She has not had any definitive treatment for the complaint. On examination she was found to be healthy looking, anicteric and no peripheral lymphadenopathy. Abdominal examination revealed a swelling in the right upper quadrant and extending to the peri-umbilical region. The roughly oval swelling measuring 12 $\mathrm{cm}$ in its greatest dimension was mobile in the horizontal plane. It was not possible to get above the swelling. There were no another abnormalities. A clinical diagnosis of mesenteric cyst was made and a contrast abdominal computerized tomography (CT) scan of the abdomen was performed.

The contrast CT scan revealed a large lobulated soft mass measuring $13.9 \times 8.3 \mathrm{~cm}$ in the transverse mesocolon. The mass was isoattenuating in density relative to muscle, enhanced intravenous contrast and displaced bowel loops. Similar masses of varying sizes ranging from $1.3 \times 0.9-2.7 \times 1.7 \mathrm{~cm}$ were noted in the mesocolon. Arterial supply was from a branch of the right hepatic artery. The gall bladder was distended with peri-cholecystic fluid. The liver, spleen and kidneys were reported normal. Hematological and biochemical parameters including liver function test and alpha-fetoprotein were within normal limits

Patient had laparotomy and a multilobulated mass measuring $13 \mathrm{~cm}$ in its greatest dimension attached to the gallbladder and posterioinferior surface of the liver was noted [Fig.1,2]. It was also adherent to the omentum, mesocolon and duodenum. An excision of the mass and cholecystectomy was done. The histopathology of excised mass revealed focal nodular hyperplasia [Fig.3,4].
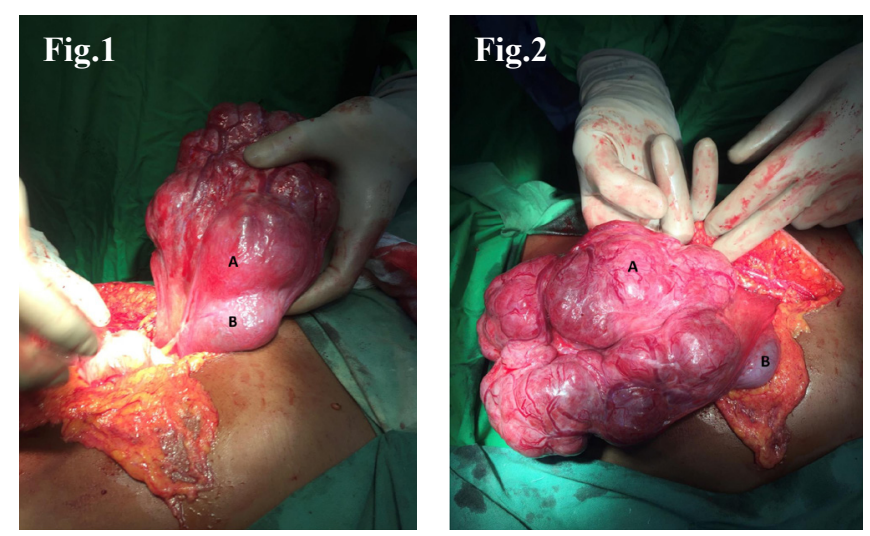

Fig.1,2(a): Mass attached to the gall bladder and edge of the liver. (b): gall bladder.

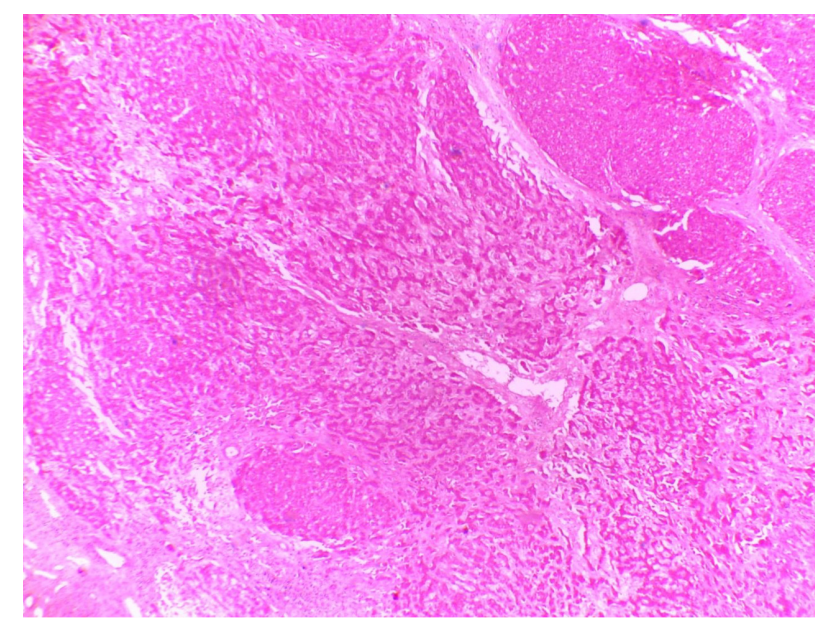

Fig.3: Histological section showing liver tissue with abnormal artitecture, hepatocytes nodules surrounded by fibrous septa and ductular proliferation ( $H \& E \times 40)$.

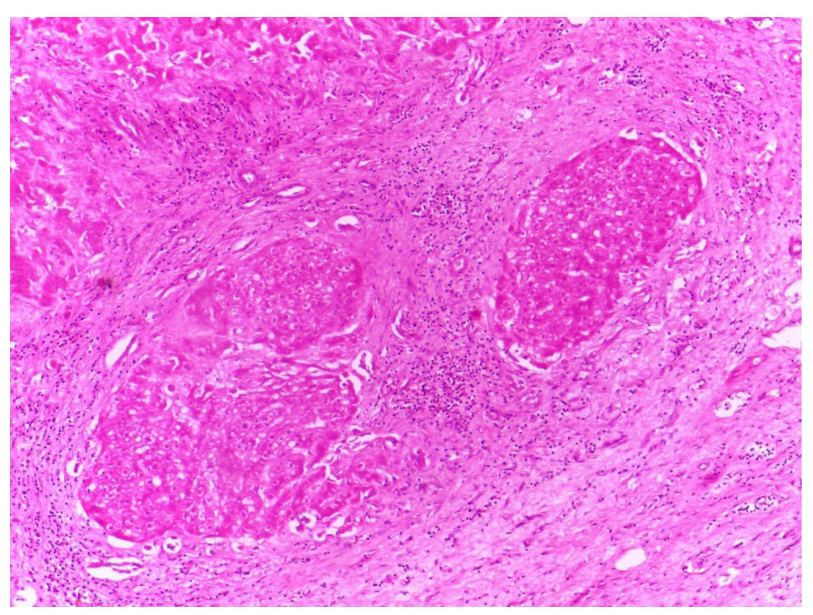

Fig.4: Higher magnification of liver tissue in fig.1 showing nodules with inflamed fibrous septa $(H \& E \times 100)$. 


\section{Discussion}

Focal nodular hyperplasia (FNH) accounts for $8 \%$ of all primary hepatic tumors with an estimated prevalence of $0.9 \%$ in the general population [3]. The term describes an entity of lobular proliferation of normally differentiated hepatocytes, frequently around a central fibrovascular scar [4]. Previously considered to be a harmatoma, FNH is now generally accepted to be a hyperplastic response to hyper-perfusion by the characteristic anomalous arteries found in the center of these nodules [5]. Pregnancy and oral contraceptives have not been demonstrated to play a role in development or progression of FNH [6]. FNH is most often solitary ( 80 to 95 percent), and usually less than $5 \mathrm{~cm}$ in diameter. Only 3 percent are larger than $10 \mathrm{~cm}$, although FNH as large as $19 \mathrm{~cm}$ have been reported $[4,7]$.

FNH is seen in both sexes and throughout the age spectrum, although it is found predominantly in women (in a ratio of 8 or 9:1) between the ages of 20 and 50 years [5]. FNH comprises up to 2 percent of liver tumors in children [8]. The characteristics of the typical FNH patient have changed as a result of the widespread availability and application of modern cross-sectional imaging. Frequently now, the lesion is picked up incidentally through high-resolution imaging performed for unrelated clinical reasons but can also present with vague abdominal symptoms due to mass effect causing pain and bloatedness. It can present with smaller lesions and atypical features in men. It is rarely symptomatic and once a definitive diagnosis has been established, active treatment is almost never required [1]. The natural history of FNH is one of stability and lack of complications. However, it should be recognized that symptomatic FNH does occur, as well as FNH behaving in an unusual fashion such as rapid growth. Both of these findings are indications for resection. The mechanisms by which FNH lesions may cause pain are unclear. However, there are well-documented cases of intra-lesional hemorrhage [4] and rupture [9]. In some patients (mainly those with large nodules), FNH causes vague, non-specific abdominal pain related to pressure exerted on Glisson's capsule. It can also present as dyspepsia, feelings of fullness or epigastric discomfort due to pressure on adjacent organs.

Radiological imaging is invaluable in diagnosing FNH. FNH may be definitively diagnosed without histopathology in circumstances in which specific characteristic features are present on multi-phase CT and contrast-enhanced MRI. In CT, a lesion exhibiting homogeneous late arterial phase enhancement with a hypo-dense central scar is diagnostic of FNH. Contrastenhanced MRI scanning has been shown to be the most sensitive modality for characterizing this lesion [10]. Compared with abdominal gray-scale ultrasonography or CT, MRI has a high diagnostic yield for FNH, with sensitivity of $68-70 \%$ and specificity of $98-100 \%$ [11-13]. Particularly, MRI with hepatobiliary-specific contrast agents, such as gadoxetic acid and gadobenate dimeglumine is very useful in diagnosing $\mathrm{FNH}$, and it has been reported to have $96-97 \%$ specificity and $96 \%$ positive predictive value $[13,14]$.

With the advances in modern hepatobiliary imaging most cases of FNH can be diagnosed with reasonable certainty nonetheless, there will always be cases in which diagnostic certainty is not achieved. Accordingly, in patients where diagnostic certainty is not achieved, resection can be justified in asymptomatic patients to avoid the undertreatment of occult malignancy. Other options include biopsy with histological analysis and conservative management with repeated imaging. The objective of lesion biopsy is to achieve a definite diagnosis and hence avoid surgery for benign lesions. It is noteworthy that biopsy can result in diagnostic inaccuracy due to sampling errors. Furthermore, the difficulty of distinguishing large FNH lesions from well differentiated or fibrolamellar HCC on biopsy histology has been wellreported [15]. The second issue concerns the risk 
for needle track seeding of tumor cells from what may be a potentially operable malignant lesion. There are several published algorithms, which advise that where diagnostic doubt exists after imaging, percutaneous biopsy should be avoided in operable disease [2]. Systematic follow-up remains the gold standard in asymptomatic patients with FNH [1]. However elective surgery should be considered in symptomatic patients, in those with marked enlargement and in case of uncertainty of diagnosis. Resection is now the modern treatment for symptomatic FNH and has been associated with low morbidity and very good post-resection quality of life.

\section{Conclusion}

Symptomatic FNH can be safely resected with excellent rates of morbidity and zero mortality. Alternatively, patients presenting with asymptomatic definitively diagnosed FNH can be safely managed conservatively. Contrast-enhanced MRI scanning has been shown to be the most sensitive modality for characterizing this lesion.

Contributors: AOO: manuscript writing, patient management; OSB, TOO: manuscript editing, patient management; KBB: critical inputs into the manuscript and histopathology. AOO will act as guarantor. All authors approved the final version of this manuscript.

Funding: None; Competing interests: None stated.

\section{References}

1. Perrakis A, Vassos N, Grutzmann R, Croner RS. What is changing in indications and treatment of focal nodular hyperplasia of the liver. Is there any place for surgery? Ann Hepatol. 2107;16:333-341.

2. Navavro AP, Gomez D, Lamb CM, Brooks A, Cameron IC. Focal nodular hyperplasia: a review of current indications for and outcome of hepatic resection. HPB (Oxford). 2014;16:503-511.

3. Vilgrain V. Focal nodular hyperplasia. Eur J Radiol. 2006;58:236-245.

4. Nguyen BN, Flejou JF, Terris B, Belghiti J, Degott C.
Focal nodular hyperplasia of the liver: a comprehensive pathologic study of 305 lesions and recognition of new histologic forms. Am J Surg Pathol. 1999;23:1441-1454.

5. Wanless IR, Mawdsley C, Adams R. On the pathogenesis of focal nodular hyperplasia of the liver. Hepatology. 1985;5:1194.

6. Rifai K, Mix H, Krusche S, PotthoffA, Manns MP, Gebel MJ. No evidence of substantial growth progression or complications of large focal nodular hyperplasia during pregnancy. Scand J Gastroenterol. 2013;48:88-92.

7. Craig J, Peters R, Edmundson H. Tumors of the liver and intrahepatic bile ducts, Fasc 26, $2^{\text {nd }}$ ed, DC Armed Forces Institute of Pathology, Washington, DC 1989. pp.6.

8. Reymond D, Plaschkes J, Lüthy AR, Leibundgut K, Hirt A, Wagner HP. Focal nodular hyperplasia of the liver in children: review of follow-up and outcome. J Pediatr Surg. 1995;30:1590-1593.

9. Behrend M, Flemming P, Halbfass HJ. Spontaneous bleeding of focal nodular hyperplasia as a rare cause of acute abdomen. Chirurg. 2001;72:1201-1204.

10. Cherqui D, Rahmouni A, Charlotte F, Boulahdour H, Metreau JM, Meignan M, et al. Management of focal nodular hyperplasia and hepatocellular adenoma in young women: a series of 41 patients with clinical, radiological, and pathological correlations. Hepatology. 1995;22:1674-1681.

11. Assy N, Nasser G, Djibre A, Beniashvili Z, Elias S, Zidan J. Characteristics of common solid liver lesions and recommendations for diagnostic workup. World $\mathrm{J}$ Gastroenterol. 2009;15:3217-3227.

12. Hussain SM, Terkivatan T, Zondervan PE, Lanjouw E, de Rave S, Ijzermans JN, et al. Focal nodular hyperplasia: findings at state-of-the-art MR imaging, US, CT, and pathologic analysis. Radiographics. 2004;24:3-17.

13. Bieze M, van den Esschert JW, Nio CY, Verheij J, Reitsma JB, Terpstra V, et al. Diagnostic accuracy of MRI in differentiating hepatocellular adenoma from focal nodular hyperplasia: prospective study of the additional value of gadoxetate disodium. AJR Am J Roentgenol. 2012;199:26-34.

14. Fowler KJ, Brown JJ, Narra VR. Magnetic resonance imaging of focal liver lesions: approach to imaging diagnosis. Hepatology. 2011;54:2227-2237

15. Hanaoka J, Shimada M, Utsunomiya T, Imura S, Morine $\mathrm{Y}$, Ikemoto $\mathrm{T}$, et al. Huge focal nodular hyperplasia difficult to distinguish from well-differentiated hepatocellular carcinoma. Hepatol Res. 2012;42:727731. 